\title{
SMB, AGLA und das Statin-Conundrum
}

\section{Michel Romanens, \\ Franz Ackermann \\ Vascular Risk Foundation VARIFO}

Eine Stellungnahme des Swiss Medical Board findet sich im Anschluss an diesen Artikel auf S. 1788
Metaanalysen der CTT-Autoren zeigen konsistent, dass pro 1,0 mmol/1 LDL-Senkung mit einem Statin eine relative Risikosenkung für Herzinfarkt (tödlich oder nicht tödlich) von $22 \%$ in 10 Jahren resultiert [1]. Da $2 / 3$ sämtlicher Herzinfarkte gemäss PROCAMStudie bei Personen mit einem Risiko von weniger als $20 \%$ in 10 Jahren auftreten [2], stellt sich die Frage, ob die Statine bei Personen ohne hohes Risiko (definiert als Herzinfarktrisiko von $>20 \%$ in 10 Jahren) eingesetzt werden sollen. In seinem Bericht von April 2014 beantwortet das Swiss Medical Board (SMB) diese Frage eindeutig mit «Nein» und akzeptiert somit a priori, dass die Mehrzahl der Herzinfarkte «schicksalhaft» auftreten. Nach der Intervention durch die Arbeitsgruppe Lipide und Atherosklerose (AGLA) [3] ist nun für das SMB statt erst ab ESC 10\% schon ab einem ESC-Risiko von 7,5\% (reines Sterberisiko) eine Statinbehandlung grundsätzlich zu erwägen [4], obwohl effiziente Prävention gemäss der CTT-Studie 2012, auf welche sich das SMB stark beruft, bereits ab einem Risiko von ESC 1,7\% nachgewiesen wurde [5]. Diese Diskussion betrifft keine Banalitäten: Gemäss eigenen Berechnungen der Stiftung Vascular Risk Foundation VARIFO beträgt der 10-Jahres-Exzess für vermeidbare Koronar-
Auf andere Aspekte der Nutzenberechnung wie «number needed to treat» oder «return on investment» tritt das SMB erst gar nicht ein und schliesst auch den Kosteneffekt der Statine betreffend vermiedener Koronareingriffe und den damit verbundenen sozialen Kosten aus. Mit dieser Haltung stellt das SMB insgesamt eine strenge Statinindikation bei Gesunden, während die meisten Empfehlungen ein Herzinfarktrisiko (tödlich und nicht tödlich) ab 7,5\%10,0\% behandeln würden (z. B. CTT low risk metaanalysis 2012, AGLA 2012, ATP III panel 2014, NICE 2014), eine Situation, in der praktisch niemand mehr für ein Statin qualifiziert und die Obsoleszenz der Risikorechner bewirkt wird [8].

Die weltweit kontrovers geführte Diskussion um die «richtige» Indikation für Statine bei Gesunden wird seit über 10 Jahren intensiv geführt, denn Statinstudien stellen mathematische Aggregate dar, daraus abgeleitete Risiken sind im Einzelfall statistisch gesehen überhaupt nie signifikant (Risiko 0\% bis $100 \%$ ), es handelt sich hier also um ein therapeutisches Conundrum [9]. Dies bedeutet: Man kann statistisch signifikante Statineffekte zwar auf der Populationsebene, nicht aber auf der Individualebene beobachten.
Korrespondenz: Dr. med. Michel Romanens Ziegelfeldstrasse 1 $\mathrm{CH}-4600$ Olten

info[at]kardiolab.ch

\section{Das Swiss Medical Board muss genauer definieren, was eine «klinisch manifeste Arteriosklerose» ist.}

eingriffe hochgerechnet für 1,929 Millionen Versicherte im Alter zwischen 51 und 70 Jahren zwischen 5632 und 6068, für vermeidbare Herzinfarkte 2816 bis 3034 und für vermeidbare koronare Todesfälle 939 bis 1011, wenn wir die AGLA-Richtlinien mit den SMB-Richtlinien vergleichen [6].

Ferner erwägt das SMB ethische und rechtliche Fragen, insbesondere wird moniert, dass Statine bekannte Nebenwirkungen haben, bei der Behandlung gesunder Personen könnten sich deswegen sogar Haftpflichtfragen stellen. Die Kosten-Nutzen-Berechnungen sind für das SMB anhand von QALYs zudem für Statine prohibitiv hoch, so dass aus der Sicht des SMB gesunde Personen kaum für präventive Statinbehandlungen qualifizieren sollten, doch das SMB rechnet auch hier falsch [7]. Als Nicht-Gesunde werden im vom SMB publizierten definitiven Bericht (7/2014) explizit Personen mit «Diabetes mellitus, genetischen Dyslipidämien und manifesten arteriosklerotischen Veränderungen» genannt.
Betrachten wir deshalb nun ein Individuum, einen wegen Brustbeschwerden «zur kardiologischen Beruhigung» zugewiesenen 60-jährigen Giessereiarbeiter, seit über 10 Jahren Nichtraucher, unbehandelter Blutdruckmittelwert 110/75 mm Hg, LDL 3,2 mmol/l, HDL 2,0 mmol/l, kein Diabetes mellitus Typ 2 bekannt. Die berechneten 10-Jahres-Risiken betragen für dieses Individuum gemäss ESC 0,9\%.

Die Messung des Arterienalters mittels Ultraschall [10] mit einer validierten Methode (Total Plaque Area TPA) [11] dokumentiert nach 5 Minuten Untersuchungsdauer eine schwere, fortgeschrittene Karotisatheromatose eines 78-Jährigen (Abb. 1). Auf Nachfrage schildert der Patient eine Episode vor einem Jahr, die mit einer TIA mit heteronymer Hemianopsie anamnestisch sehr gut vereinbar ist. Zudem zeigte die transthorakale Sonographie des Aortenbogens eine teilweise mobile Plaque IV. Grades $(25 \times 10 \mathrm{~mm})$.

Wie eingangs erwähnt, treten $>70 \%$ der Herzinfarkte bei Gesunden im Nicht-Hochrisikobereich 


\section{Abbildung 1}

Prognostisch relevante Karotisatheromatose (total plaque area $40 \mathrm{~mm}^{2}$ ) bei einem 60 -jährigen Patienten mit niedrigem kardiovaskulärem Risiko. Aufgrund sämtlicher Plaque beträgt das Arterienalter 78 Jahre [16].
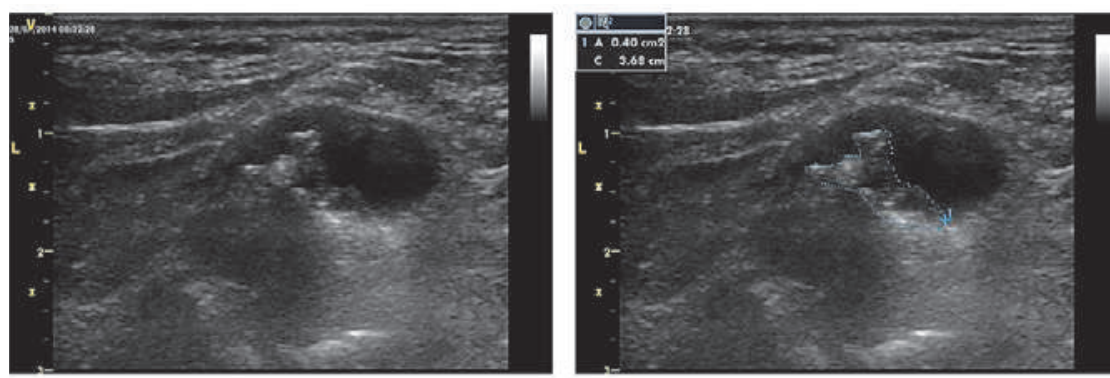

auf, die also ein AGLA-Risiko von $<20 \%$ in 10 Jahren aufweisen (Zudem: $1 / 3$ der Herzinfarkte treten bei einem AGLA-Risiko $<10 \%$ auf).

Aufgrund der Nachtest-Wahrscheinlichkeit für Herzinfarkt [12] in 10 Jahren errechnet sich bei diesem Patienten ein NNT von 26 (Framingham NNT 12), falls das LDL auf $1,8 \mathrm{mmol} / 1$ gesenkt wird, ferner ein «return on investment» von 1,50 Franken für AGLA und von 3 Franken für Framingham. Aus Sicht der Prävention zeigt dieses Beispiel, dass die Zusatzinformation aus der Karotisatheromatose eine Situation erkennen lässt, bei welcher der Statineinsatz bei Tageskosten von 0,51 Franken (80 mg Atorva / 2) kosteneffizient ist [13]. Selbstverständlich ist bei diesem Patienten wegen der von ihm bisher nicht mitgeteilten TIA vor einem Jahr ohnehin eine intensive medikamentöse Prävention indiziert.

Der Patient hat eine mit Ultraschall in 5 Minuten quantifizierte, «manifeste Arteriosklerose». Damit ist auch eine weitere Prämisse des SMB erfüllt. Die Lösung des Conundrums, und danach richten sich in unserer Region in Olten mittlerweile eine Vielzahl von Hausärztinnen und Hausärzten, liegt also darin, ein Statin zu rezeptieren, wenn eine Atheromatose nachweisbar und anhand der Berechnung der Nachtest-Wahrscheinlichkeit ausreichend hoch ist. Bei Personen mit niedrigerem Risiko in den NachtestBerechnungen würde eine Statinbehandlung allerdings unterbleiben, da diese keine «manifeste Arteriosklerose» vorzuweisen haben. Im Zweifelsfall kann die Bestimmung der Karotisatheromatose nach einem Jahr wiederholt werden, auch kann der Effekt präventiver Massnahmen auf die Karotisatheromatose im Individuum beobachtet werden [14]. Damit wird die Haupttodesursache in der Schweiz die Atherosklerose - quantifizierbar und die Statinbehandlung aus dem Conundrum der Aggregatmedizin in den Einzelfall projiziert und damit individualisiert.

Das Swiss Medical Board muss deshalb nun genauer definieren, was eine «klinisch manifeste Arte- riosklerose» ist. Denn in der Schweiz werden auch mit präventiver Fragestellung Bildgebungsverfahren angewendet, welche die Atherosklerose sichtbar machen. Aus der Sicht des Klinikers bleibt auch im niedrigen Risikobereich gemäss den Europäischen Richtlinien eine Primärprävention mit Statinen eine Notwendigkeit, wenn eine relevante Atherosklerose mittels Bildgebung dokumentiert ist [15]

\section{Literatur}

1 Cholesterol Treatment Trialists. Collaboration. Efficacy and safety of more intensive lowering of LDL cholesterol: a meta-analysis of data from 170000 participants in 26 randomised trials. Lancet. 2010;376:1670-81. Cholesterol Treatment Trialists' Ctt Collaborators. The effects of lowering LDL cholesterol with statin therapy in people at low risk of vascular disease: meta-analysis of individual data from 27 randomised trials. Lancet. 2012;6736:1-10.

2 www.assmann-stiftung.de/wp-content/uploads/2013/06/ Hamburg_16-05-2009_web4.pdf

3 www.saez.ch/docs/saez/2014/2930/de/SAEZ-02687.pdf

4 www.saez.ch/docs/saez/2014/2930/de/SAEZ-02833.pdf

5 In der CTT 2012-Arbeit steht: «The present results show that reduction of LDL cholesterol with statin therapy significantly reduced the risk of major vascular events in individuals with 5-year risk lower than 10\% (in whom the mean risks were $2,6 \%$ for major coronary events plus $3 \%$ for other major vascular events), even in those with no previous history of vascular disease, diabetes, or chronic kidney disease (panel).» Folglich für 10 Jahre: $2,6 \% \times 2=5,2 \%$ MACE-Risiko, ESC-Risiko = 5,2\% / $3=$ $1,73 \%$.

6 http://physicianprofiling.ch/VEMSLetterSMB082014.pdf

7 Das Szenario des SMB für die QALY-Berechnungen ist allerdings irreführend. Siehe dazu die Berechnungen unter: http://physicianprofiling.ch/StatineSMB.xls

8 http://physicianprofiling.ch/VEMSLetterSMB082014.pdf

9 www.cardioexchange.org/voices/the-risk-predictionconundrum-individual-risk-vs-population-risk/

10 Romanens M, Ackermann F, Sudano I, Szucs T, Spence JD. Arterial age as a substitute for chronological age in the AGLA risk function could improve coronary risk prediction. Swiss Med Wkly. 2014;144:w13967. www.smw.ch/content/smw-2014-13967/

11 www.ncbi.nlm.nih.gov/pubmed/23393396/www.ncbi. nlm.nih.gov/pubmed/22222422 / www.ncbi.nlm.nih. gov/pubmed/21311059 / www.ncbi.nlm.nih.gov/ pubmed/21311059 / www.ncbi.nlm.nih.gov/ pubmed/12468791 / www.ncbi.nlm.nih.gov/ pubmed/21653216

12 http://scopri.ch/posttestcalculators1.htm

13 www.varifo.ch/Varifo2014.xls

14 Spence JD, Hackam D. Treating Arteries Instead of Risk Factors. A Paradigm Change in Management of Atherosclerosis. Stroke. 2010;41(6):1193-9.

15 http://eurheartj.oxfordjournals.org/content/33/13/1635. full.pdf

16 www.smw.ch/content/smw-2014-13967 